RM3-TH/03-2

Roma, $1351 / 03$

SHEP-02-34

\title{
Coupling of the light vector meson to the vector and to the tensor current
}

\author{
D. Becirevic ${ }^{a, b}, \mathrm{~V} \cdot \mathrm{Lubicz}^{c}, \mathrm{~F} \cdot$ Mescia $^{d}$ And C. $^{\text {Tarantino }}{ }^{c}$ \\ ${ }^{a}$ Laboratoire de Physique Théorique (Bât 210) ${ }^{1}$, Université de Paris Sud, \\ Centre d'Orsay, 91405 Orsay-Cedex, France. \\ ${ }^{b}$ Dip. di Fisica, Università di Roma "La Sapienza", \\ Piazzale Aldo Moro 2, I-00185 Rome, Italy. \\ ${ }^{c}$ Dip. di Fisica, Univ. di Roma Tre and INFN, Sezione di Roma III, \\ Via della Vasca Navale 84, I-00146 Rome, Italy. \\ ${ }^{d}$ Department of Physics and Astronomy, University of Southampton, \\ Southampton SO171BJ, United Kingdom.
}

\begin{abstract}
We present results for the coupling of the light vector mesons to the tensor current, relative to the standard vector meson decay constants. From an $\mathcal{O}(a)$-improved lattice study, performed at three values of the lattice spacing in the quenched approximation, our final values (in the continuum limit), in the $\overline{\mathrm{MS}}$ scheme at $\mu=2 \mathrm{GeV}$, are: $\left.f_{\rho}^{T} / f_{\rho}=0.72(2){ }_{-0}^{+2}\right), f_{K^{*}}^{T} / f_{K^{*}}=0.74(2), f_{\phi}^{T} / f_{\phi}=0.76(1)$.
\end{abstract}

\section{Introduction}

\subsection{Motivation for this computation}

The most direct way to extract one of the least known CKM parameters, $\left|V_{u b}\right|$, is from studies of the corresponding leptonic and semileptonic decays of $B$-mesons. Although the leptonic decays are still beyond the reach of the present experiments, CLEO, Belle and BaBar are providing rather accurate measurements of the branching ratios for the semileptonic $B \rightarrow \rho(\pi) \ell \nu$ modes [1]. On the theoretical side, lattice QCD and light cone QCD sum rules (LCSR) are expected to provide model independent information about shapes and absolute values of the relevant form factors. That necessitates good control over

\footnotetext{
${ }^{1}$ Unité mixte de Recherche du CNRS - UMR 8627.
} 
$B \rightarrow \rho l v$

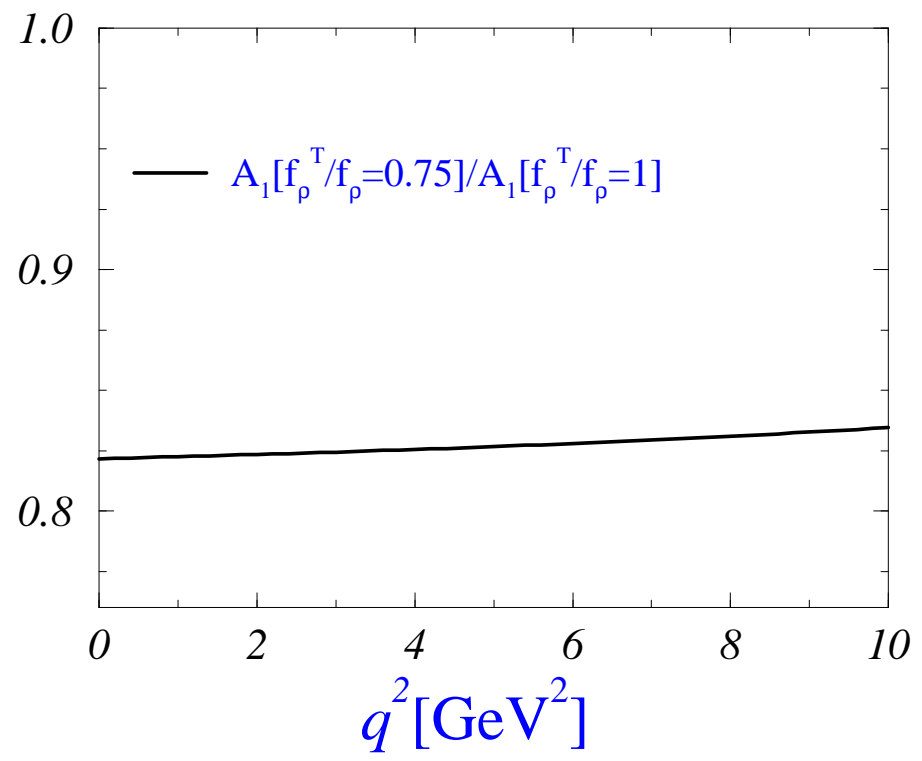

Figure 1: The effect of replacing $f_{\rho}^{T} / f_{\rho}=1 \rightarrow 0.75$ in eq. (1) results in about $15-20 \%$ suppression of the form factor $A_{1}^{B \rightarrow \rho}\left(q^{2}\right)$. The ratio is evaluated by using the leading twist distribution amplitudes from ref. [5].

the low energy (non-perturbative) QCD dynamics. The complicating feature of heavy $\rightarrow$ light decays is that the $q^{2}$-region accessed by these decays is large, e.g. for $B \rightarrow \rho \ell \nu$, with $\ell=e, \mu$, it is $0 \leq q^{2} \leq\left(m_{B}-m_{\rho}\right)^{2} \equiv 20.3 \mathrm{GeV}^{2}$. Lattice QCD results are available for large momentum transfers, $q^{2} \geq 10 \mathrm{GeV}^{2}$ (see refs. [2]), while LCSR results are expected to be reliable in the region of low $q^{2}$ 's $[3-5]$. In order to reduce the uncertainties in the form factors obtained in the latter approach, one needs better control over the nonperturbative parameters which are explicitly present in the LCSR, such as moments of the light cone wave functions, various hadronic couplings etc. A step in this direction is made in this letter, where we report results for the ratio of the coupling constants of the light vector mesons to the tensor $\left(f_{V}^{T}\right)$ and to the vector current $\left(f_{V}\right)$, which is an important ingredient in the LCSR expressions for the $B \rightarrow \rho$ and $B \rightarrow K^{*}$ semileptonic form factors. While the constant $f_{V}$ can in principle be extracted from experiments on $e^{+} e^{-} \rightarrow V^{0}$ and $\tau^{-} \rightarrow V^{-} \nu_{\tau}$, the coupling $f_{V}^{T}$ can be estimated only theoretically. To do so, we will use the lattice QCD. The first computation of this coupling has been attempted in ref. [6]. The QCDSF collaboration has also presented their preliminary results in ref. [7]. Here we will restrict our attention to the ratio of the couplings $f_{V}^{T} / f_{V}$, use the data generated at three values of the lattice spacing and extrapolate to the continuum limit. Before we enter into details of the lattice computations, let us make a brief assessment of the importance of having an accurate determination of $f_{V}^{T} / f_{V}$. 


\subsection{How important are the couplings $f_{V}$ and $f_{V}^{T}$ ?}

To exemplify the importance of having a good handle on $f_{V}^{T} / f_{V}$, we give the LCSR expression for the form factor which dominates the $B \rightarrow \rho \ell \nu$ decay rate, namely [4]

$$
\begin{aligned}
& A_{1}\left(q^{2}\right)=\frac{m_{b} f_{\rho} \mathrm{e}^{\left(m_{B}^{2}-m_{b}^{2}\right) / M^{2}}}{m_{B}^{2} f_{B}\left(m_{B}+m_{\rho}\right)} \int_{u_{0}}^{1} \frac{d u}{u} \mathrm{e}^{(1-1 / u)\left(q^{2}-m_{b}^{2}-u m_{\rho}^{2}\right) / M^{2}} \times \\
& \left\{\frac{m_{b}^{2}+u^{2} m_{\rho}^{2}-q^{2}}{2 u} \frac{f_{\rho}^{T}}{f_{\rho}} \phi_{T}(u)+\frac{m_{b} m_{\rho}}{2}\left[\int_{0}^{u} d v \frac{\phi_{\|}(v)}{1-v}+\int_{u}^{1} d v \frac{\phi_{\|}(v)}{v}\right]\right\},
\end{aligned}
$$

where $M^{2}$ is the so-called Borel parameter. The lower limit in the integral, $u_{0}$, contains information about the energy of a threshold, $s_{0}$, above which quark-hadron duality is assumed. Using the input parameters of ref. [4], at $q^{2}=0$ one has $u_{0} \simeq 0.65$, whereas for $q^{2}=10 \mathrm{GeV}^{2}$ it is $u_{0} \simeq 0.5$. The objects under integrals are the light cone distribution amplitudes, which are the functions of $u$, the fraction of the longitudinal momentum of the meson carried by one of the meson's valence quarks. Indices $T$ and $\|$ denote the polarization states of the $\rho$-meson. In the asymptotic limit, $\mu \rightarrow \infty$, these functions are $\phi_{T, \|}^{\text {asymp. }}(u)=6 u(1-u)[3]$. QCD corrections to $\phi_{T, \|}^{\text {asymp. }}(u)$ are conveniently accounted for by expanding these functions in the basis of Gegenbauer polynomials and then by computing the first few moments. In order to make the above sum rule (1) as simple as possible we did not include radiative nor higher twist corrections. They can be found in ref. [5].

The first term in eq. (1), which is numerically dominant, is proportional to $f_{\rho}^{T} / f_{\rho}$. To show the importance of that ratio we first set it to one, as has been done in [3], and then to $f_{\rho}^{T} / f_{\rho} \approx 0.75$, as typically found in recent QCD sum rule analyzes $[8,9]$. We plug those values in eq. (1), make the ratio of the two cases and plot it in fig. 1. From that plot and by varying the parameters as in ref. [4], we see that the form factor $A_{1}\left(q^{2}\right)$ gets shifted by 15-20\% . Therefore a reliable estimate of $f_{\rho}^{T} / f_{\rho}$, as to reduce the overall uncertainty on $A_{1}\left(q^{2}\right)$, is important.

\section{Definitions. Lattice details. Direct results}

\subsection{Vector meson couplings}

For a generic vector meson $V$, with valence quark content $\left(\bar{q}^{\prime} q\right)$, the couplings $f_{V}$ and $f_{V}^{T}(\mu)$ are defined through the following matrix elements

$$
\begin{aligned}
& \left\langle 0\left|\bar{q}(0) \gamma^{\mu} q^{\prime}(0)\right| V(p, \lambda)\right\rangle=f_{V} m_{V} e_{\lambda}^{\mu} \\
& \left\langle 0\left|\left(\bar{q}(0) \sigma^{\mu \nu} q^{\prime}(0)\right)(\mu)\right| V(p, \lambda)\right\rangle=i f_{V}^{T}(\mu)\left(e_{\lambda}^{\mu} p^{\nu}-e_{\lambda}^{\nu} p^{\mu}\right),
\end{aligned}
$$

where $p$ and $e_{\lambda}^{\mu}$ are the momentum and the polarization vectors. These definitions refer to Minkowski space where we take $\sigma^{\mu \nu}=(i / 2)\left[\gamma^{\mu}, \gamma^{\nu}\right]$. Notice that since the anomalous dimension of the tensor current is different from zero, the coupling $f_{V}^{T}(\mu)$ depends on the scale at which the corresponding current is renormalized. 


\subsection{Relevant correlation functions}

To estimate the values of the coupling $f_{V}^{T}(\mu)$ for various light vector mesons $V$, we will compute its ratio with $f_{V}$. This is beneficial because several systematic uncertainties, such as the dependence on the lattice spacing, are likely to cancel to a large extent. To compute $f_{V}^{T}(\mu) / f_{V}$ it is sufficient to consider the vector meson at rest $(\vec{p}=0)$ and the following ratio of correlation functions in Euclidean space (" $E$ " in the following)

$$
R(t ; \mu)=\frac{C_{T V}(t)}{C_{V V}(t)}=\frac{\left\langle\sum_{\vec{x}} \hat{T}_{0 i}(x ; \mu) V_{i}^{\dagger}(0)\right\rangle_{E}}{\left\langle\sum_{\vec{x}} \hat{V}_{i}(x) V_{i}^{\dagger}(0)\right\rangle_{E}},
$$

where on the r.h.s. we chose the second operator to be the vector current. Any other local operator with the quantum numbers $J^{P C}=1^{--}$would also be a good choice. The hat symbol indicates that the corresponding current has been improved and renormalized, which we discuss in the next subsection. By inserting a complete set of states and going towards large time separations between the source operators, the lowest meson state dominates and we have

$$
\left.R(t ; \mu)\right|_{t \gg 0}=\frac{\sum_{\lambda} e_{\lambda, i}^{*}\left\langle 0\left|\hat{T}_{0 i}(\mu)\right| V(0, \lambda)\right\rangle_{E}}{\sum_{\lambda} e_{\lambda, i}^{*}\left\langle 0\left|\hat{V}_{i}\right| V(0, \lambda)\right\rangle_{E}} .
$$

In eq. (4), we have Euclidean operators, whereas the couplings, as defined in eq. (2), refer to Minkowski space (" $M$ "). Using the fact that

$$
T_{0 i}^{E}=\left(i \bar{q}(x) \sigma_{0 i} q(x)\right)^{E} \rightarrow\left(\bar{q}(x) \sigma^{0 i} q(x)\right)^{M}, \quad V_{i}^{E}=\left(\bar{q}(x) \gamma_{i} q(x)\right)^{E} \rightarrow-i\left(\bar{q}(x) \gamma^{i} q(x)\right)^{M},
$$

it becomes clear that the plateau in the ratio (3), (i.e. eq. (4)) gives $R(\mu)=f_{V}^{T}(\mu) / f_{V}$.

\subsection{Improvement and renormalization}

Our lattice study is made by using non-perturbatively $\mathcal{O}(a)$ improved Wilson fermions, where $a$ stands for the lattice spacing. The improvement of the operators considered in this letter, $\hat{T}_{0 i}(\mu)$ and $\hat{V}_{i}$, is made through [10]

$$
\begin{aligned}
& \hat{T}_{0 i}^{E}(\mu)=Z_{T}^{(0)}(\mu a)\left(1+b_{T} a m_{q}\right)\left[T_{0 i}^{E}(a)+c_{T}(a) \partial_{0} V_{i}(a)\right], \\
& \hat{V}_{i}^{E}=Z_{V}^{(0)}(a)\left(1+b_{V} a m_{q}\right)\left[V_{i}^{E}(a)-c_{V}(a) \partial_{0} T_{0 i}^{E}(a)\right] .
\end{aligned}
$$

$c_{T, V}(a)$ ensure that bare currents computed on the lattice are free of $\mathcal{O}(a)$ lattice artifacts; the constants $Z_{V}^{(0)}(a)$ and $Z_{T}^{(0)}(\mu a)$ provide the matching to the continuum local operators and also their renormalization in the chiral limit, whereas the quark mass dependent 
artifacts of $\mathcal{O}\left(a m_{q}\right)$ are subtracted by setting the coefficients $b_{V}(a)$ and $b_{T}(a)$ to their values determined non-perturbatively and by using boosted perturbation theory, respectively. The numerical values of all of the above constants are listed in table 1, where we also give the main features of our lattices ${ }^{1}$.

\begin{tabular}{|c|c|c|c|}
\hline$\beta=6 / g_{0}^{2}$ & 6.0 & 6.2 & 6.4 \\
\hline$c_{S W}[13]$ & 1.769 & 1.614 & 1.526 \\
\hline$L^{3} \times T$ & $16^{3} \times 52$ & $24^{3} \times 64$ & $32^{3} \times 70$ \\
\hline \# conf. & 500 & 200 & 150 \\
\hline$\kappa_{1}$ & 0.1335 & 0.1339 & 0.1347 \\
\hline$\kappa_{2}$ & 0.1338 & 0.1344 & 0.1349 \\
\hline$\kappa_{3}$ & 0.1340 & 0.1349 & 0.1351 \\
\hline$\kappa_{4}$ & 0.1342 & 0.1352 & 0.1353 \\
\hline$a / r_{0}[14]$ & 0.1863 & 0.1354 & 0.1027 \\
\hline$c_{T}[15]$ & 0.07 & 0.06 & 0.05 \\
\hline$c_{V}[15]$ & -0.11 & -0.09 & -0.08 \\
\hline$b_{V}[16]$ & 1.47 & 1.41 & 1.36 \\
\hline$b_{T}[17]$ & 1.23 & 1.22 & 1.21 \\
\hline$Z_{V}^{(0)}(a)[12]$ & $0.766(2)$ & $0.775(2)$ & $0.795(3)$ \\
\hline$Z_{T}^{(0)}(\mu a=1)[12]$ & $0.833(2)$ & $0.847(3)$ & $0.867(6)$ \\
\hline
\end{tabular}

Table 1: Summary of our lattice details. We also give the values of the improvement coefficients (with the corresponding references), and the values of the lattice spacing relative to the parameter $r_{0} \approx 0.5 \mathrm{fm}$.

\subsection{Direct lattice results}

Information about our lattices is provided in table 1. Our data is obtained at three lattice spacings. The improvement ensures that the effects linear in lattice spacing are not present

\footnotetext{
${ }^{1}$ Please note that our lattice data refer to the same runs as those used in refs. [11,12], where additional information can be found.
} 
and therefore the extrapolation to the continuum limit $(a \rightarrow 0)$ is expected to be smoother. In table 2 we collect results directly obtained for our lattices.

\begin{tabular}{c|c|c|c|c|c|}
\cline { 2 - 5 } \multicolumn{1}{c|}{$\beta=6.0$} & $\underline{\kappa_{1}}$ & $\underline{\kappa_{2}}$ & $\underline{\kappa_{3}}$ & $\underline{\kappa_{4}}$ \\
\cline { 2 - 6 } & $m_{P} / m_{V}$ & $0.743(6)$ & $0.709(8)$ & $0.682(10)$ & $0.650(12)$ \\
$f_{V}^{T} / f_{V}$ & $0.739(5)$ & $0.733(7)$ & $0.730(10)$ & $0.728(14)$ \\
\cline { 2 - 6 } & $\beta=6.2$ \\
$m_{P} / m_{V}$ & $0.807(4)$ & $0.751(7)$ & $0.661(11)$ & $0.575(16)$ \\
$f_{V}^{T} / f_{V}$ & $0.761(4)$ & $0.744(5)$ & $0.731(9)$ & $0.736(15)$ \\
\hline$m_{P} / m_{V}$ & $0.750(8)$ & $0.707(9)$ & $0.648(11)$ & $0.563(15)$ \\
$f_{V}^{T} / f_{V}$ & $0.747(4)$ & $0.737(5)$ & $0.728(7)$ & $0.723(15)$ \\
\hline
\end{tabular}

Table 2: Ratio of the masses of the pseudoscalar and vector mesons consisting of two degenerate quarks of mass corresponding to $\kappa_{i}$ specified in table 1 . We also give the values of the $f_{V}^{T} / f_{V}$ ratio for each $\kappa_{i}$, where the renormalization scale is set to $\mu=(1 / a)_{\beta}$.

In forming the ratio (3) we used time reversal to symmetrise the correlation functions as

$$
\begin{aligned}
& C_{T V}(t) \rightarrow \frac{1}{2}\left[C_{T V}(t)-C_{T V}(T-t)\right], \\
& C_{V V}(t) \rightarrow \frac{1}{2}\left[C_{V V}(t)+C_{V V}(T-t)\right],
\end{aligned}
$$

where $t=0,1, \ldots T / 2$. On the plateaus, the symmetrised correlator produces the usual tanh-factor, and thus the final fit form is

$$
R(t ; 1 / a)=\frac{f_{V}^{T}}{f_{V}} \tanh \left[m_{V}(T / 2-t)\right] .
$$

In improving the currents (see eq. (6)) we use the symmetric discrete derivative i.e. $\partial_{0} g(t)=$ $[g(t+1)-g(t-1)] / 2$, where $g(t)$ is the generic Green function computed on the lattice.

Finally, the fit is made using the same intervals with which the vector meson masses have been extracted (see ref. [11]), namely

$$
\begin{aligned}
& \beta=6.0 \rightarrow t \in[11,23], \\
& \beta=6.2 \rightarrow t \in[12,28], \\
& \beta=6.4 \rightarrow t \in[17,28] .
\end{aligned}
$$

The results are listed in table 2 , and an illustration of the ratios $R(t ; 1 / a)$ is provided in fig. 2 . 

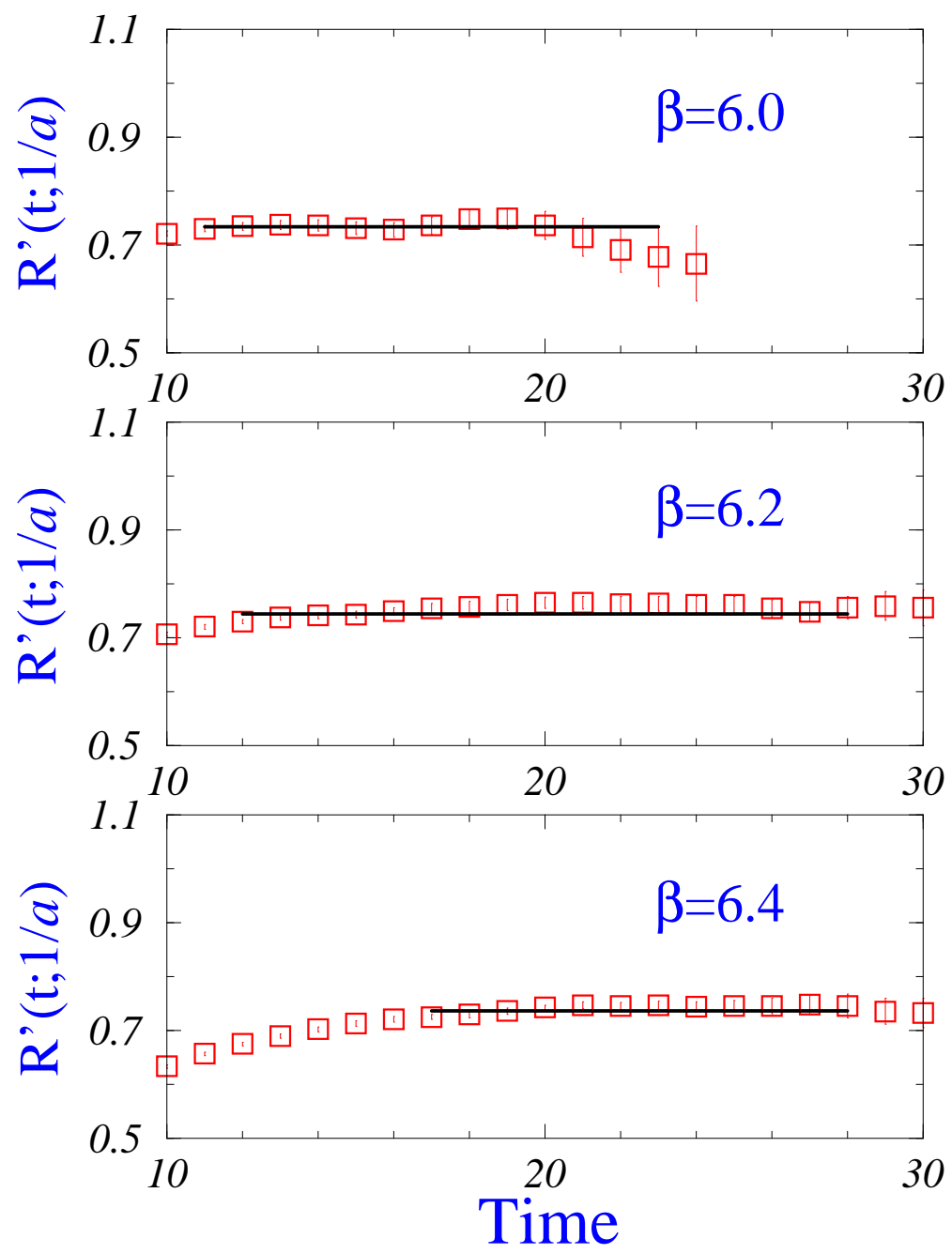

Figure 2: Ratio $R^{\prime}(t ; 1 / a)=R(t ; 1 / a) / \tanh \left[m_{V}(T / 2-t)\right]$ (see eqs. $(3,9)$ ) which on the plateau leads to $f_{V}^{T} / f_{V}$. Fit to a constant is also shown. We show the signals for all three values of the lattice spacing, and we choose $\kappa=\kappa_{2}$ specified in table 1 for each $\beta$. 


\section{Physical results}

\section{$3.1 \quad$ Running}

We first need to run all the ratios from the scale $\mu=1 / a$ to the conventional $\mu=2 \mathrm{GeV}$. In our computation we used the constant $Z_{T}(\mu)$ determined non-perturbatively in the (Landau)RI/MOM renormalization scheme [12] which, up to NLO in perturbation theory, is the same scheme as $\overline{\mathrm{MS}}(\mathrm{NDR})$. The scale dependence of the tensor bilinear, and thus of $Z_{T}(\mu)$, is obtained by solving the renormalization group equation at NLO:

$$
\frac{d \log \left\langle T_{\mu \nu}(\mu)\right\rangle}{d \log \mu}=-\gamma_{T}\left(\alpha_{s}(\mu)\right)
$$

where the anomalous dimension at NLO in perturbation theory reads [18]

$$
\gamma_{T}\left(\alpha_{s}(\mu)\right)=\gamma_{T}^{(0)} \frac{\alpha_{s}(\mu)}{4 \pi}+\gamma_{T}^{(1)}\left(\frac{\alpha_{s}(\mu)}{4 \pi}\right)^{2}=\frac{8}{3} \frac{\alpha_{s}(\mu)}{4 \pi}+\frac{4}{27}\left(543-26 n_{F}\right)\left(\frac{\alpha_{s}(\mu)}{4 \pi}\right)^{2} .
$$

At the same order, the running coupling is governed by

$$
\frac{d \alpha_{s}(\mu)}{d \log \mu}=-2 \beta_{0} \alpha_{s}(\mu)-\frac{\beta_{1}}{2 \pi} \alpha_{s}^{2}(\mu)
$$

with the beta function coefficients being $\beta_{0}=11-2 n_{F} / 3$, and $\beta_{1}=102-38 n_{F} / 3$. Since $f_{V}^{T}(\mu)$ inherits the scale dependence from the tensor operator, it is completely equivalent to solve eq. (11) in terms of $f_{V}^{T}(\mu)$ instead of the matrix element $\left\langle T_{\mu \nu}(\mu)\right\rangle$. We finally have

$$
\begin{aligned}
& f_{V}^{T}(\mu)=f_{V}^{T}\left(\mu_{0}\right) W\left[\mu, \mu_{0}\right] \\
& \equiv f_{V}^{T}\left(\mu_{0}\right)\left(\frac{\alpha_{s}(\mu)}{\alpha_{s}\left(\mu_{0}\right)}\right)^{4 /\left(33-2 n_{F}\right)} \times\left[1+J_{T} \frac{\alpha_{s}(\mu)-\alpha_{s}\left(\mu_{0}\right)}{4 \pi}\right], \\
& \text { where } \quad J_{T}=\frac{\gamma_{T}^{(1)} \beta_{0}-\gamma_{T}^{(0)} \beta_{1}}{2 \beta_{0}^{2}}=\frac{2}{9} \frac{12411-1260 n_{F}+52 n_{F}^{2}}{\left(33-2 n_{F}\right)^{2}} .
\end{aligned}
$$

Since we work in the quenched approximation, we set $n_{F}=0$, and we run to $\mu=2 \mathrm{GeV}$ by using $\Lambda_{\overline{\mathrm{MS}}}^{n_{F}=0}=0.250(25) \mathrm{GeV}$. The values of the lattice spacing are computed by using the conservative estimate $a_{\beta=6.0}^{-1}=2.0(1) \mathrm{GeV}$. The other $a_{\beta}^{-1}$ are related to $a_{\beta=6.0}^{-1}$ by using the ratios of $a / r_{0}$ [14]. The net effect is that

$$
W\left[2 \mathrm{GeV}, a^{-1}\right]=\left.1.000(2)(0)\right|_{\beta=6.0},\left.1.010(2)(1)\right|_{\beta=6.2},\left.1.019(2)(1)\right|_{\beta=6.4},
$$

where the first error comes from the $5 \%$ variation of $a_{\beta=6.0}^{-1}$, and the second one from $10 \%$ uncertainty on $\Lambda_{\frac{n_{F}}{\mathrm{MS}}}=0$. As we can see those uncertainties are a few per mil and are thus negligible. 


\section{$3.2 \quad f_{\rho}^{T}(2 \mathrm{GeV}) / f_{\rho}, f_{K^{*}}^{T}(2 \mathrm{GeV}) / f_{K^{*}}$ and $f_{\phi}^{T}(2 \mathrm{GeV}) / f_{\phi}$}

To get the physically relevant results (corresponding to $V=\rho, K^{*}, \phi$ ), we fit our results to the form

$$
\frac{f_{V}^{T}(2 \mathrm{GeV})}{f_{V}}=\alpha_{0}+\alpha_{1}\left(a m_{P}\right)^{2}+\alpha_{2}\left(a m_{P}\right)^{4},
$$

where $\left(a m_{P}\right)$ stands for the pseudoscalar meson mass that is computed by using the same quarks (non-degenerate in mass) as the ones used to compute the ratio on the l.h.s. (see table (2)). As our central values, we chose to quote the results obtained through the linear fit $\left(\alpha_{2}=0\right)$, whereas the quadratic fit is used to assess systematic uncertainties. The physical results, corresponding to $V=\rho, K^{*}, \phi$, are obtained by choosing $\left(a m_{P}\right)^{2}=$ $\left(a m_{\pi}\right)^{2},\left(a m_{K}\right)^{2},\left[2\left(a m_{K}\right)^{2}-\left(a m_{\pi}\right)^{2}\right]$, respectively. The values of $\left(a m_{\pi}\right)^{2}$ and $\left(a m_{K}\right)^{2}$ are obtained from the fit of our data to

$$
\left(a m_{V}\right)=\delta_{0}+\delta_{1}\left(a m_{P}\right)^{2}
$$

and the intersection of this form with the curve $\left(a m_{V}\right)=r \sqrt{\left(a m_{P}\right)^{2}}$, where $r=5.6,1.8$, i.e. $\left(m_{\rho} / m_{\pi}\right)_{\text {phys. }}$, and $\left(m_{K^{*}} / m_{K}\right)_{\text {phys. }}$, respectively. This extrapolation-interpolation to physical mesons is shown in fig. 3 and the results are presented in table 3. Our final results are obtained through a linear extrapolation to the continuum limit $(a \rightarrow 0)$, i.e.

$$
\left(\frac{f_{V}^{T}(2 \mathrm{GeV})}{f_{V}}\right)(a)=\left(\frac{f_{V}^{T}(2 \mathrm{GeV})}{f_{V}}\right)(0)+\mathcal{B}\left(a / r_{0}\right)^{2},
$$

where on the l.h.s. we use our results as obtained at three values of the lattice spacing, the values of $\left(a / r_{0}\right)$ [14] on the r.h.s. are given in table 1 , and $\mathcal{B}$ is a fit parameter. Notice that since our calculation is improved at $\mathcal{O}(a)$, we omit the term linear in $a$ when extrapolating to the continuum limit. The continuum extrapolation is illustrated in fig. 4 and our final results are

$$
\begin{aligned}
f_{\rho}^{T}(2 \mathrm{GeV}) / f_{\rho} & =0.720(24)\left(\begin{array}{l}
+16 \\
-0
\end{array}\right), \\
f_{K^{*}}^{T}(2 \mathrm{GeV}) / f_{K^{*}} & =0.739(17)\left(\begin{array}{l}
+3 \\
-0
\end{array}\right), \\
f_{\phi}^{T}(2 \mathrm{GeV}) / f_{\phi} & =0.759(9)(0) .
\end{aligned}
$$

\subsection{A brief discussion of the systematic uncertainties}

Many systematic uncertainties cancel in the ratio of the vacuum-to-meson couplings computed here. For example, the uncertainty due to the lattice spacing is only present in the evolution of the tensor current from the renormalization scale $\mu=1 / a$, down to $\mu=2 \mathrm{GeV}$. As we showed, that uncertainty is at the level of a few per mil, thus negligible.

Our central results at each value of $\beta$, presented in table 3 , are obtained by fitting our data to the form (16) linearly $\left(\alpha_{2}=0\right)$. The second errors reflect the difference 

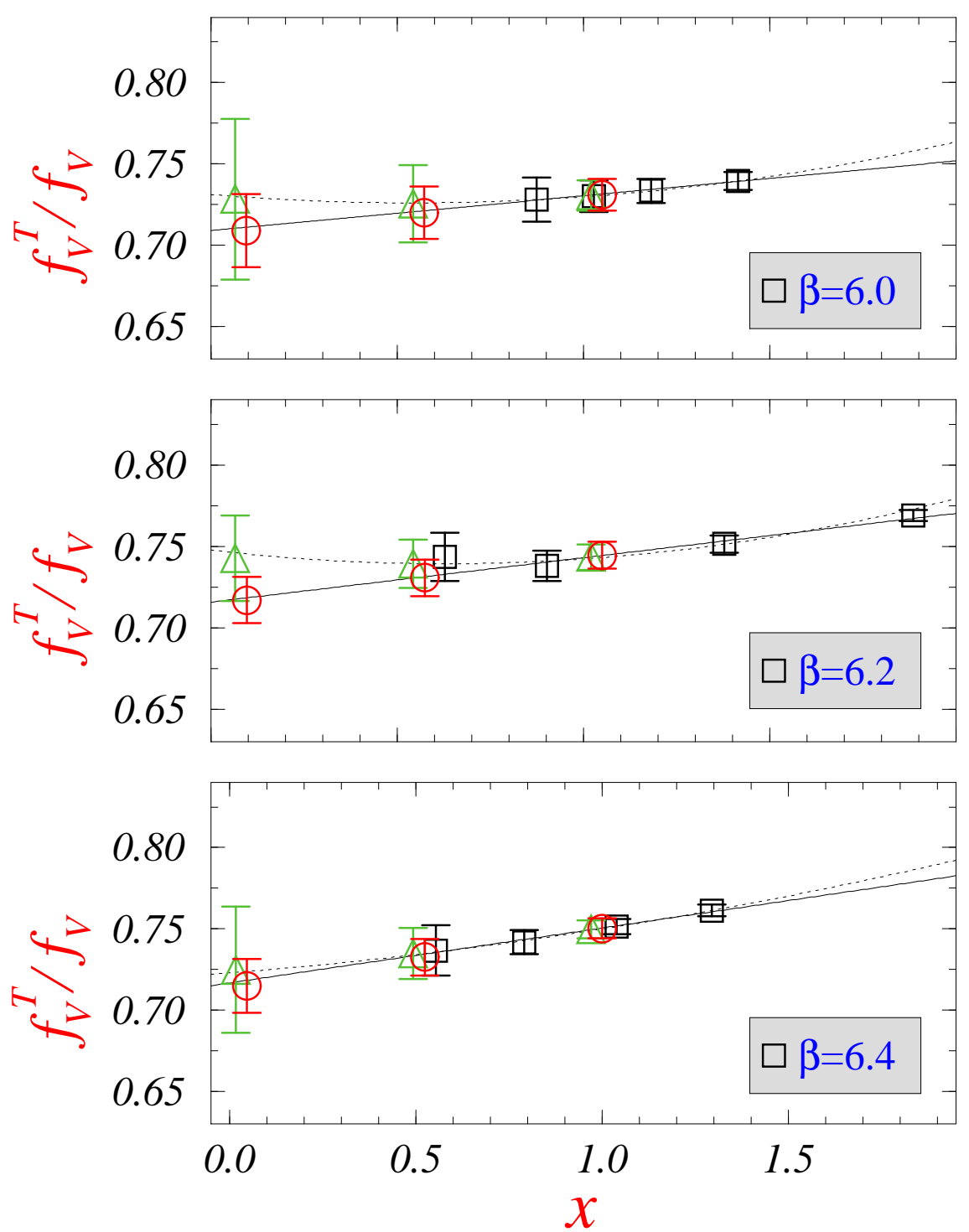

Figure 3: Ratios $f_{V}^{T}(2 \mathrm{GeV}) / f_{V}$ that are directly accessed in our lattice study (denoted by squares) are fit linearly (solid line) and quadratically (dotted line) according to eq. (16). To make the abscissa dimensionless we defined $x=\left(a m_{P}\right)^{2} /\left(a m_{s s}\right)^{2}$, where $\left(a m_{s s}\right)^{2}=2\left(a m_{K}\right)^{2}-\left(a m_{\pi}\right)^{2}$. Resulting ratios for the $\rho, K^{*}$ and $\phi$ mesons are marked by circles. The ratios obtained from the quadratic fit (gray circles) are slightly shifted to the left as to make them discernible from the ones obtained through the linear fit (red circles).From left to right they correspond to $\rho, K^{*}$ and $\phi$ mesons. 


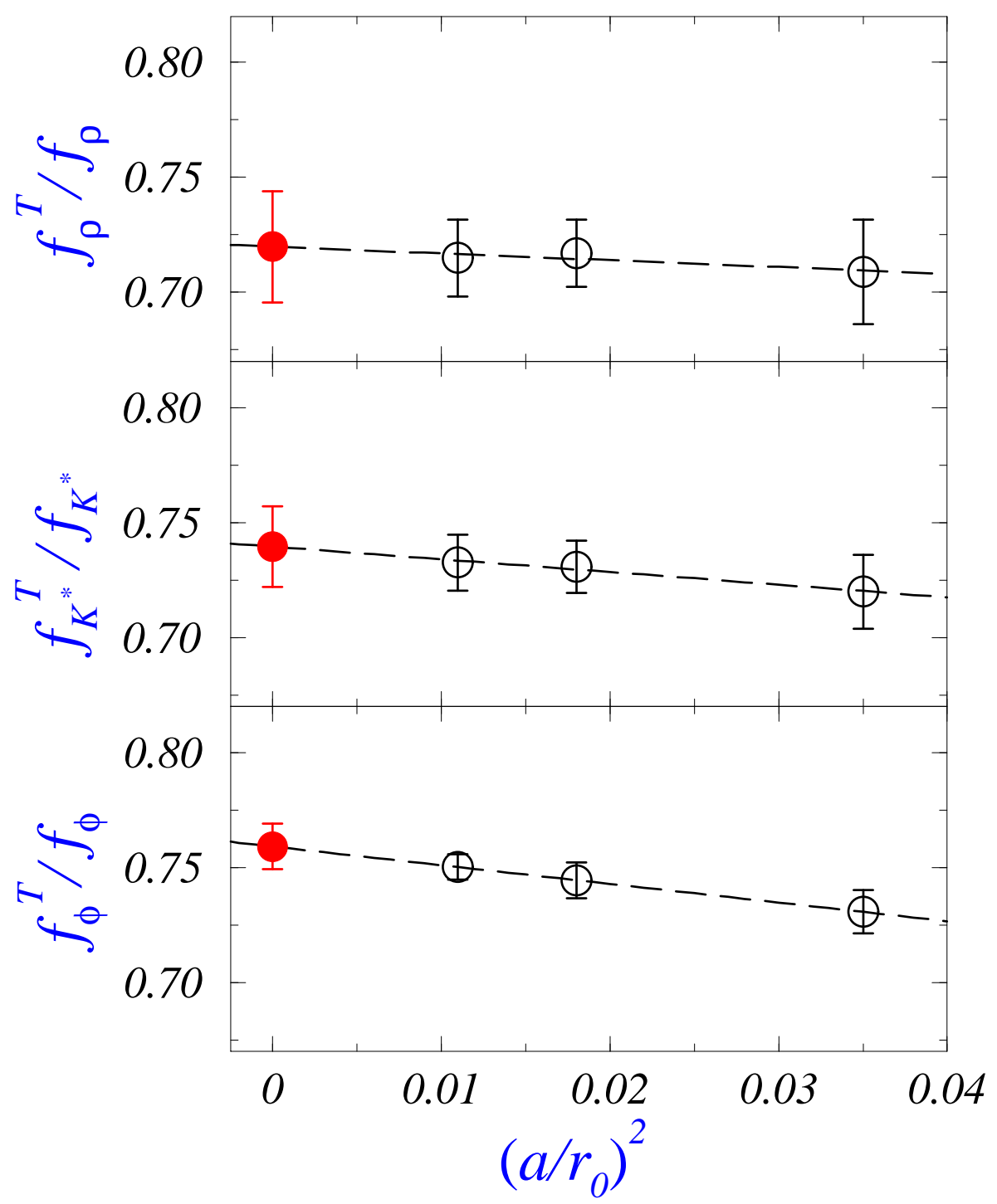

Figure 4: Extrapolation to the continuum limit $(a \rightarrow 0)$. 


\begin{tabular}{|c|c|c|c|}
\hline$\left(a / r_{0}\right)^{2}$ & $f_{\rho}^{T}(2 \mathrm{GeV}) / f_{\rho}$ & $f_{K^{*}}^{T}(2 \mathrm{GeV}) / f_{K^{*}}$ & $f_{\phi}^{T}(2 \mathrm{GeV}) / f_{\phi}$ \\
\hline \hline 0.035 & $0.708(23)\left(_{-00}^{+19}\right)$ & $\left.0.720(16){ }_{-0}^{+6}\right)$ & $0.731(10)(0)$ \\
0.018 & $0.717(14)\left(_{-0}^{+25}\right)$ & $0.731(11)\left(_{-0}^{+8}\right)$ & $0.744(8)(0)$ \\
0.011 & $0.715(17)\left(_{-0}^{+10}\right)$ & $0.733(11)\left(_{-0}^{+2}\right)$ & $0.750(6)(0)$ \\
\hline
\end{tabular}

Table 3: The values of the ratio of the vacuum-to-meson couplings mediated by the tensor vs. vector current, renormalized at $\mu=2 \mathrm{GeV}$ in the (Landau)RI/MOM scheme, which at NLO in perturbation theory is the same as the $\overline{\mathrm{MS}}(\mathrm{NDR})$ renormalization scheme.

between those and the values obtained through the quadratic fit (16). These errors are quite significant for the $\rho$-meson because that meson is reached through an extrapolation. On the other hand, they are small for the $K^{*}$ - and $\phi$-mesons.

The systematic errors induced by the presence of the chiral logarithms in extrapolating to the chiral limit are unlikely to affect our ratios because the same hadron state appears in both the numerator and the denominator. One may also speculate that for the same reason quenching effects should be small, but we prefer to consider our results as quenched and leave the unquenching issue to future lattice studies.

In the continuum extrapolation we use a linear fit in the square of the lattice spacing since our results do not suffer from the $\mathcal{O}(a)$ artefacts. In fact all the renormalisation and improvement constants are known non-perturbatively, except for $b_{T}$ which is obtained from 1-loop boosted perturbation theory. Since the residual $\mathcal{O}(a)$-effect is $\propto a m_{q} \alpha_{s}^{2}$ and we work with the light quarks $\left(\left(a m_{q}\right) \leq 0.07[11]\right)$, this can be safely ignored.

\section{Tensor coupling and the comparison with other the- oretical predictions}

To get the absolute values of the tensor couplings we will now use our ratios from eq. (19), and multiply them by the physical values for $f_{\rho, K^{*}, \phi}$ extracted from experiments, i.e.

$$
f_{\rho, K^{*}, \phi}^{T}(2 \mathrm{GeV})=\left(\frac{f_{\rho, K^{*}, \phi}^{T}(2 \mathrm{GeV})}{f_{\rho, K^{*}, \phi}}\right)^{\text {latt. }} f_{\rho, K^{*}, \phi}^{\text {exp. }}
$$




\subsection{Our results for $f_{\rho, K^{*}, \phi}^{T}(2 \mathrm{GeV})$ and comparison with other lat- tice calculations}

The experimental values for the charged vector meson couplings $f_{\rho^{ \pm}}, f_{K^{* \pm}}$ can be extracted from the data for $\tau$-lepton decays [21]:

$$
B R\left(\tau \rightarrow \rho^{-} \nu_{\tau}\right)=25.0(3) \%, \quad B R\left(\tau \rightarrow K^{*-} \nu_{\tau}\right)=1.29(5) \%
$$

By using the tree level expression

$$
B R\left(\tau \rightarrow V^{-} \nu_{\tau}\right)=\frac{G_{F}^{2} m_{\tau}\left|V_{q q^{\prime}}\right|^{2}}{8 \pi} \tau_{\tau} m_{V}^{2} f_{V}^{2}\left(1-\frac{m_{\tau}^{2}}{2 m_{V}^{2}}\right)\left(1+\frac{m_{V}^{2}}{m_{\tau}^{2}}\right)^{2}
$$

and $\left|V_{u d}\right|=0.9735,\left|V_{u s}\right|=0.220, \tau_{\tau}=290.6 \mathrm{ps}, m_{\tau}=1.777 \mathrm{GeV}, m_{K^{*}}=0.892 \mathrm{GeV}$, and $G_{F}=1.1664 \cdot 10^{-5} \mathrm{GeV}^{-2}$, all taken from ref. [21], we obtain

$$
f_{\rho^{ \pm}}^{\text {exp. }} \simeq 208 \mathrm{MeV}, \quad f_{K^{* \pm}}^{\text {exp. }} \simeq 217 \mathrm{MeV}
$$

where we display only the central values. Notice that the value of $f_{\rho^{ \pm}}$is consistent with $f_{\rho^{0}}=216(5) \mathrm{MeV}$, obtained from $e^{+} e^{-}$-annihilation.

The constant $f_{\phi}$ can be obtained from the width $\Gamma_{\phi^{0} \rightarrow e^{+} e^{-}}=1.32(4) \mathrm{keV}$, which we combine with $\alpha_{\text {em. }}^{-1}=137.036, m_{\phi}=1.019 \mathrm{GeV}[21]$ and get $^{2}$

$$
f_{\phi}^{\exp .}=\left(\frac{27 m_{\phi}}{4 \pi \alpha_{\mathrm{em}}^{2}} \Gamma_{\phi^{0} \rightarrow e^{+} e^{-}}\right)^{1 / 2} \simeq 233 \mathrm{MeV} .
$$

We then insert the above values for $f_{\rho, K^{*}, \phi}^{\exp }$ in (20) and arrive at our estimate for the tensor couplings:

$$
\begin{aligned}
& f_{\rho^{ \pm}}^{T}(2 \mathrm{GeV})=150(5)\left(\begin{array}{l}
+3 \\
-0
\end{array}\right) \mathrm{MeV}=152(7) \mathrm{MeV}, \\
& f_{K^{* \pm}}^{T}(2 \mathrm{GeV})=160(4)\left(\begin{array}{l}
+1 \\
-0
\end{array}\right) \mathrm{MeV}=161(4) \mathrm{MeV}, \\
& f_{\phi}^{T}(2 \mathrm{GeV})=177(2)(0) \mathrm{MeV}=177(2) \mathrm{MeV} .
\end{aligned}
$$

The result for $f_{\rho}^{T}(2 \mathrm{GeV})$ agrees also very well with the estimate of ref. [7] in which this constant has been computed on the lattice, at three values of the lattice spacing, in the quenched approximation. In that study the perturbatively evaluated renormalisation constant has been used. Their new calculation [19] includes also the non-perturbative determination of $Z_{T}(\mu)$, and after extrapolating to the continuum limit they quote

$$
f_{\rho}^{T}(2 \mathrm{GeV})=150(4) \mathrm{MeV} .
$$

Although the systematic uncertainty of that result is still preliminary, we note a very pleasant agreement with our value given in eq. $(25)^{3}$.

\footnotetext{
${ }^{2}$ In the previous version of this paper, eqs. (22) and (24) contained typos. Numerical results however were correct. We thank Patricia Ball for drawing our attention to this.

${ }^{3}$ We are indebted to Gerrit Schierholz for pointing out the ref. [7] to us and for communicating their new results.
} 


\subsection{Comparison with the QCD sum rule results}

The QCD sum rule (QSR) estimates [5, 8] are made at the renormalization scale $\mu=1 \mathrm{GeV}$. We run those values to $\mu=2 \mathrm{GeV}$, by using eq. (14) and $\Lambda_{\frac{n_{F}}{\mathrm{MS}}}^{n^{2}}=338(40) \mathrm{MeV}$ [20], i.e. $f_{V}^{T}(2 \mathrm{GeV})=0.9667(6) \times f_{V}^{T}(1 \mathrm{GeV})$. Thus the QSR estimates are

$$
\begin{gathered}
f_{\rho}^{T}(2 \mathrm{GeV})=155 \pm 10 \mathrm{MeV}, \\
f_{K^{*}}^{T}(2 \mathrm{GeV})=179 \pm 10 \mathrm{MeV}, \\
f_{\phi}^{T}(2 \mathrm{GeV})=208 \pm 15 \mathrm{MeV} .
\end{gathered}
$$

Note also that the coupling $f_{\rho}^{T}(\mu)$ has been re-examined in ref. [9], essentially confirming the results of ref. [8]. By comparing the lattice results (25) with the QSR values (27), we see that the agreement of the two methods for $f_{\rho}^{T}$ is very good, quite good for $f_{K^{*}}^{T}$ and less good for $f_{\phi}^{T}$. Investigation of the source for that discrepancy is beyond the scope of the present paper. It should, however, be stressed that the LCSR yield results for full QCD while our results are quenched and therefore a perfect match is anyway not expected.

\section{Summary}

In this letter we presented quenched lattice results for the ratio of the couplings of the light vector mesons to the tensor and to the vector currents. Those ratios enter the LCSR analyses of the phenomenologically important heavy $\rightarrow$ light meson semileptonic decay form factors. From the results obtained with $\mathcal{O}(a)$ improved Wilson quarks, and with high statistics data, we were able to extrapolate to the continuum limit. The resulting ratios are then multiplied by the experimentally measured vector meson couplings to arrive at the tensor couplings. Comparison with the prediction obtained from the QCD sum rule analysis indicates good agreement for $f_{\rho, K^{*}}^{T}(2 \mathrm{GeV})$, whereas the agreement is not good in the case of $f_{\phi}^{T}(2 \mathrm{GeV})$. Our result for $f_{\rho, K^{*}}^{T}(2 \mathrm{GeV})$ also agrees very well with the lattice estimate of refs. $[7,19]$.

\section{Acknowledgements}

We thank G. Martinelli and J. Flynn for reading the manuscript, as well as Patricia Ball and G. Schierholz for correspondance. This work has been partially supported by M.U.R.S.T, and by the E.C.'s contract HPRN-CT-2000-00145 "Hadron Phenomenology from Lattice QCD”. 


\section{References}

[1] B. H. Behrens et al. [CLEO], Phys. Rev. D 61 (2000) 052001, [hep-ex/9905056]; B. Aubert et al. [BABAR], [hep-ex/0207080], Y. Kwon [Belle], ICHEP-02.

[2] J.M. Flynn et al. [UKQCD Collaboration], Nucl. Phys. B 461 (1996) 327 [hep-ph/9506398]; A. Abada, D. Becirevic, P. Boucaud, J.M. Flynn, J.P. Leroy, V. Lubicz and F. Mescia [SPQcdR collaboration], hep-lat/0209116.

[3] V. L. Chernyak and A. R. Zhitnitsky, Phys. Rept. 112 (1984) 173.

[4] A. Ali, V. M. Braun and H. Simma, Z. Phys. C 63 (1994) 437 [hep-ph/9401277]; P. Ball and V. M. Braun, Phys. Rev. D 55 (1997) 5561 [hep-ph/9701238].

[5] P. Ball and V. M. Braun, Phys. Rev. D 58 (1998) 094016 [hep-ph/9805422].

[6] D. Becirevic et al., Nucl. Phys. Proc. Suppl. 73 (1999) 222 [hep-lat/9809187]; see also [hep-lat/9809129].

[7] S. Capitani et al., Nucl. Phys. Proc. Suppl. 79 (1999) 548 [hep-ph/9905573].

[8] P. Ball and V. M. Braun, Phys. Rev. D 54 (1996) 2182 [hep-ph/9602323].

[9] A. P. Bakulev and S. V. Mikhailov, Eur. Phys. J. C 17 (2000) 129 [hep-ph/9908287]; Eur. Phys. J. C 19 (2001) 361 [hep-ph/0006206].

[10] K. Jansen et al., Phys. Lett. B 372 (1996) 275 [hep-lat/9512009].

[11] D. Becirevic, V. Lubicz and C. Tarantino [SPQ(CD)R Collaboration], hep-lat/0208003.

[12] D. Becirevic et al. [SPQcdR Collaboration], hep-lat/0209168.

[13] M. Luscher, S. Sint, R. Sommer, P. Weisz and U. Wolff, Nucl. Phys. B 491 (1997) 323 [hep-lat/9609035].

[14] S. Necco and R. Sommer, Nucl. Phys. B 622 (2002) 328 [hep-lat/0108008].

[15] T. Bhattacharya, R. Gupta, W. J. Lee and S. R. Sharpe, Phys. Rev. D 63 (2001) 074505 [hep-lat/0009038]; Nucl. Phys. Proc. Suppl. 106 (2002) 789 [hep-lat/0111001].

[16] M. Luscher, S. Sint, R. Sommer and H. Wittig, Nucl. Phys. B 491 (1997) 344 [hep-lat/9611015].

[17] S. Capitani, hep-lat/0211036 (see also references therein).

[18] J. A. Gracey, Phys. Lett. B488 (2000) 175 [hep-ph/0007171].

[19] G. Schierholz et al. [QCDSF Collaboration], in preparation. 
[20] S. Bethke, hep-ex/0211012.

[21] K. Hagiwara et al. [PDG Collaboration], Phys. Rev. D 66 (2002) 010001. 